\title{
Stochastic Stabilization of a Noisy Linear System with a Fixed-Rate Adaptive Quantizer
}

\author{
Serdar Yüksel ${ }^{1}$
}

\begin{abstract}
Zooming type adaptive quantizers have been introduced in the networked control literature as efficient coders for stabilizing open-loop unstable noise-free systems connected over noiseless channels with arbitrary initial conditions. Such quantizers can be regarded as a special class of the Goodman-Gersho adaptive quantizers. In this paper, we provide a stochastic stability result for such quantizers when the system is driven by an additive noise process. Conditions leading to stability are evaluated when the system is driven by noise with non-compact support for its probability measure. It is shown that zooming quantizers are efficient and almost achieve the fundamental lower bound of the logarithm of the absolute value of an unstable eigenvalue. In particular, such quantizers are asymptotically optimal when the unstable pole of the linear system is large for a weak form of stability.
\end{abstract}

\section{INTRODUCTION}

We consider a remote stabilization problem where a controller which has access to quantized measurements acts on a plant, which is open-loop unstable. Before proceeding further with the description of the system, we discuss the quantization policy investigated.

First, let us define a quantizer. A quantizer, $Q$, for a scalar continuous variable is a mapping from $\mathbb{R}$ to a finite set, characterized by corresponding bins $\left\{\mathcal{B}_{i}\right\}$ and their representation $\left\{q^{i}\right\}$, such that $\forall i, Q(x)=q^{i}$ if and only if $x \in \mathcal{B}_{i}$.

Of particular interest is the class of uniform quantizers. A uniform quantizer: $Q^{\Delta}: \mathbb{R} \rightarrow \mathbb{R}$ with step size $\Delta$ and an (even) $K$ number of levels satisfies the following for $k=1,2 \ldots, K$ :

$$
Q^{\Delta}(x)=\left\{\begin{array}{c}
\left(-\frac{K}{2}+k-1 / 2\right) \Delta, \text { if } \\
x \in\left[\left(-\frac{K}{2}+k-1\right) \Delta,\left(-\frac{K}{2}+k\right) \Delta\right) \\
\left(\frac{K}{2}+1 / 2\right) \Delta, \text { if } x \geq \frac{K}{2} \Delta \\
-\left(\frac{K}{2}+1 / 2\right) \Delta, \text { if } x \leq-\frac{K}{2} \Delta
\end{array}\right.
$$

A general class of quantizers are those which are adaptive. Let $\mathbb{S}$ be a set of states for a quantizer state

\footnotetext{
${ }^{1}$ Mathematics and Engineering Program and the Department of Mathematics and Statistics, Queen's University, Kingston, Ontario, Canada, K7L 3N6. Email: yuksel@mast.queensu.ca. Research is supported by the Natural Sciences and Engineering Research Council of Canada (NSERC)
}

$S$. Let $F: \mathbb{S} \times \mathbb{R} \rightarrow \mathbb{S}$ be a state update-function. An adaptive quantizer has the following state update equations:

$$
S_{t+1}=F\left(S_{t}, Q_{t}\left(x_{t}\right)\right)
$$

Here, $Q_{t}$ is the quantizer applied at time $t, x_{t}$ is the input to the quantizer $Q_{t}$, and $S_{t}$ is the state of the quantizer.

One particular class of adaptive quantizers is the Goodman-Gersho type quantizers [1], which also include the zooming-type quantizers [4]. One type of Goodman-Gersho adaptive quantizers has the following form with $Q^{\Delta}$ being a uniform quantizer with a given number of levels and bin-size $\Delta$ and $\bar{Q}$ determining the updates in the bin-size of the uniform quantizer:

$$
\begin{gathered}
q_{t}=Q^{\Delta_{t}}\left(x_{t}\right) \\
\Delta_{t+1}=\Delta_{t} \bar{Q}\left(x_{t}\right)
\end{gathered}
$$

Here $\Delta_{t}$ characterizes the uniform quantizer, as it is the bin size of the quantizer at time $t$.

In the following, we provide the linear system description to which the quantizer is applied.

We consider an LTI discrete-time scalar system described by

$$
x_{t+1}=a x_{t}+b u_{t}+d_{t},
$$

where $x_{t}$ is the state at time $t, u_{t}$ is the control input, and $\left\{d_{t}\right\}$ is a sequence of zero-mean independent, identically distributed (i.i.d.) random variables such that each of the random variables admits a probability distribution $\nu$ which is absolutely continuous with respect to the Lebesgue measure on $\mathbb{R}$, and for every open set $D \in \mathbb{R}$, $\nu(D)>0$. Furthermore, $E\left[d_{t}^{2}\right]<\infty$. Here $a$ is the system coefficient with $|a|>1$, that is, the system is open-loop unstable. Thus, the class of systems considered includes the case where $\left\{d_{t}\right\}$ are Gaussian random variables.

This system is connected over a noiseless channel with a finite capacity to an estimator (controller). The controller has only access to the information it has received through the channel. The controller in our model estimates the state and then applies her control. As such, 
the problem reduces to a state estimation problem since such a scalar system is controllable. Hence, the stability of the estimation error is equivalent to the stability of the state itself.

\section{A. Literature Review}

Zooming type adaptive quantizers, which will be described further in the paper, have been recently introduced by Brockett and Liberzon [6], Liberzon [4] and Liberzon and Nesic [5] for the use in remote stabilization of open-loop unstable, noise-free systems with arbitrary initial conditions. Nair and Evans [10] provided a stability result under the assumption that the quantization is variable-rate and showed the first result for a noisy setup (with unbounded support for the noise probability measure) that on average it suffices to use more than $\log _{2}(|a|)$ bits to achieve a form of stability. [10] used asymptotic quantization theory to obtain a time-varying scheme, where the quantizer is used at certain intervals at a very high rate, and at other times, the quantizer is not used. In contradistinction, we provide a technique which allows us to both provide a result for the case when the quantizer is fixed-rate as well as to obtain an invariance condition for a probability measure on the quantizer parameters. There is also a vast literature on quantizer design in the communications and information theory community, as conveniently presented in the survey paper [11]. One important further reference here is the work by Goodman and Gersho [1], where an adaptive quantizer was introduced and the adaptive quantizer's stationarity properties were investigated when the source fed to the quantizer is independent and identically distributed with a finite second moment. Kieffer and Dunham [3] have obtained conditions for the stochastic stability of a number of coding schemes when the source considered is also stable, that is when it has an invariant distribution; where various forms of stability of the quantizer and the estimation error have been studied. In our case, however, the schemes in [1] and [3] are not directly applicable, as the process we consider is open-loop unstable, as well as Markovian. Another related work by Kieffer is [2].

\section{Stochastic Stability Via Adaptive QUANTIZERS: ZOOMING TYPE QUANTIZERS}

An example of Goodman-Gersho [1] type adaptive quantizers, which also has been shown to be effective in control systems, are those that have zoom level coefficients as the quantizer state [4]. In the zooming scheme, the quantizer enlarges the bin sizes in the quantizer until the state process is in the range of the quantizer, in which phase the quantizer is in the perfect-zoom phase. Due to the effect of the system noise, occasionally the state will be in the overflow region of the quantizer, leading to an under-zoom phase. We will refer to such quantizers as zooming quantizers.

In the following, we will assume the communication channel to be a discrete noiseless one with capacity $R$.

We now state our main result.

Theorem 2.1: Consider a zooming type adaptive quantizer applied to the linear control system described by (1), with the following update rules for $t \geq 0$ and with $\Delta_{0} \in \mathbb{R}$ selected arbitrarily and $\hat{x}_{-1}=0$ :

$$
\begin{gathered}
u_{t}=-\frac{a}{b} \hat{x}_{t} \\
\hat{x}_{t}=a \hat{x}_{t-1}+Q^{\Delta_{t}}\left(x_{t}\right) \\
\Delta_{t+1}=\Delta_{t} \bar{Q}\left(\left|\frac{x_{t}}{\Delta_{t} 2^{R-1}}\right|\right)
\end{gathered}
$$

If there exist $\delta, \epsilon, \eta>0$ with $\eta<\epsilon$ and $L>0$ such that,

$$
\begin{aligned}
& \bar{Q}(x) \geq|a|+\delta \quad \text { if } \quad|x|>1 \\
& \frac{|a|}{|a|+\epsilon-\eta}<\bar{Q}(x)<1 \quad \text { if } \quad 0 \leq|x| \leq 1, \Delta>L \\
& \bar{Q}(x)=1 \quad \text { if } \quad 0 \leq|x| \leq 1, \Delta \leq L
\end{aligned}
$$

with

$$
\sqrt{\frac{E\left[d_{t}^{2}\right]}{L \frac{|a|}{|a|+\epsilon-\eta} 2^{R-1}}}<\delta
$$

and

$$
R=\log _{2}(\lceil|a|+\epsilon\rceil+1),
$$

then the adaptive quantizer policy leads to the existence of a recurrent set, furthermore with

$$
\limsup _{t \rightarrow \infty} E\left[\log \left(x_{t}^{2}\right)\right]<\infty
$$

Remark: Hence, a simple quantization scheme indeed suffices for a form of stability. The additional price of such a quantizer is an additional 1 level. The rate required is close to the lower bound presented by Wong and Brockett: $\log _{2}(|a|)$ [7]. In our case, the additional 1 term is for the overflow term for the under-zoom phase. As $|a| \rightarrow \infty, \log _{2}(|a|+1)-\log _{2}(|a|)=$ $\log _{2}(1+1 /|a|) \leq \log _{2}(e) 1 /|a| \rightarrow 0$. As such, zooming quantizers are asymptotically optimal for obtaining a finite expected logarithm of the state magnitude. $\diamond$

Remark: We note that, our result above is somewhat weaker than those found by Nair and Evans [10], as our result includes a bound on the expected value of $\log \left(x_{t}^{2}\right)$, as opposed to that of $x_{t}^{2}$. However, our result uses a time-invariant rate (that is a fixed-rate). The analysis in 
[10] uses results from asymptotic quantization theory, by applying a variable-rate scheme, where a very-high rate quantizer is applied occasionally, and quantization is not applied at other times.

Remark: We note that the stability result for such a scheme requires new techniques to be used, as classical tools in Markovian stability theory will not be applicable directly. In the following section, we use a two-stage Martingale approach to prove the existence of a recurrent set.

Our second result is the following:

Theorem 2.2: Under the setup of Theorem 2.1, if furthermore, the quantizer bin sizes are such that their (base-2) logarithms are integer multiples of some scalar $s$ and $\log (\bar{Q}()$.$) take values in integer multiples of s$, where the integers taken are relatively prime (that is they share no common divisors except for 1 ), then the jointly Markov process $\left(e_{t}, \Delta_{t}\right)$ forms a positive (Harris) recurrent Markov chain, and has a unique invariant distribution.

\section{A. Proof of Theorem 2.1}

Let $e_{t}=x_{t}-\hat{x}_{t}$. We first start with the following result, the proof of which we omit.

Lemma 2.1:

$$
\begin{aligned}
& P\left(\left(e_{t}, \Delta_{t}\right) \in C \mid\left(e_{t-1}, \Delta_{t-1}\right), \ldots,\left(e_{0}, \Delta_{0}\right)\right) \\
=\quad & P\left(\left(e_{t}, \Delta_{t}\right) \in C \mid\left(e_{t-1}, \Delta_{t-1}\right)\right), \quad \forall C \in \mathcal{B}\left(\mathbb{R} \times \mathbb{R}_{+}\right)
\end{aligned}
$$

i.e. $\left(e_{t}, \Delta_{t}\right)$ is a Markov chain.

Let $h_{t}:=\frac{e_{t}}{\Delta_{t} 2^{R-1}}$. Consider the following sets:

$$
C_{e}=\{e:|e| \leq E\} \quad C_{h}=\{h:|h| \leq 1\},
$$

with $E=2^{R-1} L\left(\frac{|a|}{|a|+\epsilon-\eta}\right)$. Further, let another set be $C_{e}^{\prime}=\{e:|e| \leq F\}$, with a sufficiently large $F$ value to be derived below. We will study the expected number of time stages between visits of $\left\{\left(e_{t}, h_{t}\right)\right\}$ to $C_{e}^{\prime} \times C_{h}$. Consider the drift of the $\left(e_{t}, h_{t}\right)$ process in Figure 1: When $e_{t}, h_{t}$ are in $C_{e} \times C_{h}$, the expected drift increases both $|h|$ and $|e|$. When the $e_{t}$ process gets outside $C_{e}^{\prime}$ and $h_{t}$ outside $C_{h}$ (under-zoomed), there is a drift for $h_{t}$ towards $C_{h}$, however, $\left|e_{t}\right|$ will keep increasing. Finally, when the process hits $C_{h}$ (perfect zoom), then the process drifts towards $C_{e}^{\prime}$. There exists an upper bound on the value that $h$ can take when $e_{t}$ is inside the compact sets, by the hypothesis of the theorem. We first show that the sequence $\left\{h_{t}, t \geq 0\right\}$ visits $C_{h}$ infinitely often with probability 1 and the expected length of the excursion is uniformly bounded over all possible values of $(e, f) \in C_{e}^{\prime} \times C_{h}$. Once $C_{h}$ visited, then the estimation error decreases on average. However, unless this is met, $\left|e_{t}\right|$ keeps increasing stochastically. Let $V\left(h_{t}\right)=h_{t}^{2}$

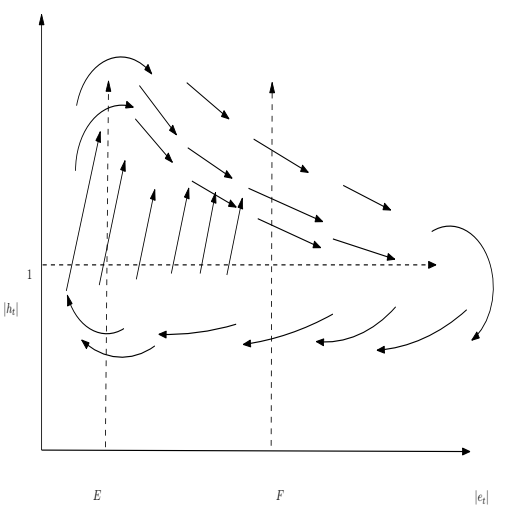

Fig. 1: Drift in the Error Process: When under-zoomed, the error increases on average; when perfectly-zoomed, the error decreases.

serve as a Lyapunov function. Define a sequence of stopping times for the perfect-zoom case with

$$
\begin{gathered}
\tau_{0}=\inf \left\{k>0:\left|h_{k}\right| \leq 1,\left|h_{0}\right| \leq 1\right\}, \\
\tau_{z+1}=\inf \left\{k>\tau_{z}:\left|h_{k}\right| \leq 1\right\}
\end{gathered}
$$

We have that, if $\left|h_{t}\right|>1$ (under-zoomed)

$$
E\left[h_{t+1}^{2} \mid h_{t}, e_{t}\right] \leq \frac{\left(a^{2}+\frac{E\left[d^{2}\right]}{\left|e_{t}\right|^{2}}\right)}{(a+\delta)^{2}}\left(h_{t}\right)^{2}
$$

Since when $\left|h_{t}\right|>1$, we have that $\left|e_{t}\right| \geq$ $2^{R-1} L\left(\frac{|a|}{|a|+\epsilon-\eta}\right)$, it follows that $E\left[h_{t+1}^{2} \mid h_{t}, e_{t}\right] \leq$ $\left(\frac{a^{2}+\frac{E\left[d^{2}\right]}{E}}{(a+\delta)^{2}}\right)\left(h_{t}\right)^{2}$. If $\left|h_{t}\right| \leq 1$, then

$$
\begin{aligned}
E\left[h_{t+1}^{2}\right] & \leq \frac{a^{2} \frac{\left(\Delta_{t}\right)^{2}}{4}+E\left[d_{t}^{2}\right]}{\left(\Delta_{t} 2^{R-1}\right)^{2}}\left(\frac{|a|+\epsilon-\eta}{|a|}\right)^{2} \\
& \leq \frac{a^{2} \frac{L^{\prime 2}}{4}+E\left[d_{t}^{2}\right]}{\left(L^{\prime} 2^{R-1}\right)^{2}}\left(\frac{|a|+\epsilon-\eta}{|a|}\right)^{2} \\
& =: K_{1},
\end{aligned}
$$

where $L^{\prime}=L \frac{|a|}{|a|+\epsilon-\eta}$ (this is a lower bound on $\Delta_{t}$ ). Hence, it follows that

$$
E\left[h_{t+1}^{2}-h_{t}^{2} \mid h_{t}, e_{t}\right] \leq-\rho h_{t}^{2}+K_{1} 1_{\left(\left|h_{t}\right| \leq 1\right)},
$$

where $1_{(U)}$ is the indicator function for event $U$ with

$$
\begin{aligned}
K_{1} & =1+\frac{a^{2} \frac{L^{\prime 2}}{4}+E\left[d_{t}^{2}\right]}{\left(L^{\prime} 2^{R-1}\right)^{2}}\left(\frac{|a|+\epsilon-\eta}{|a|}\right)^{2}, \\
\rho & =1-\frac{\left(a^{2}+\frac{E\left[d^{2}\right]}{E^{2}}\right)}{(a+\delta)^{2}}
\end{aligned}
$$

Since for two non-negative numbers $A, B>0, A^{2}+$ $B^{2} \leq(A+B)^{2}$ it follows that the hypothesis 
$\sqrt{\frac{E\left[d_{t}^{2}\right]}{L^{\prime} 2^{R-1}}}<\delta$ in the theorem statement ensures $\rho>0$. Now, define $M_{0}:=V\left(h_{0}\right)$, and for $t \geq 1$

$$
M_{t}:=V\left(h_{t}\right)-\sum_{i=0}^{t-1}\left(-\rho+K_{1} 1_{\left(h_{i} \in C_{h}\right)}\right)
$$

Hence, as $\left|h_{t}\right|>1$ when $h_{t} \notin C_{h}$, $E\left[M_{(t+1)} \mid\left(e_{s}, h_{s}\right), s \leq t\right] \leq M_{t}, \quad \forall t \geq 0$, and thus, $\left\{M_{t}\right\}$ is a Super-Martingale. Define a stopping time: $\tau^{N}=\min \left(N, \min \left\{i>0: V\left(h_{i}\right) \geq N, V\left(h_{i}\right) \leq 1,\right\}\right)$. The stopping time is bounded and the Super-Martingale sequence is also bounded. Hence, we have, by the Martingale optional sampling theorem: $E\left[M_{\left(\tau^{N}\right)}\right] \leq E\left[M_{0}\right]$. Hence, we obtain

$$
E\left[\sum_{i=0}^{\tau^{N}-1}\right] \rho \leq V\left(h_{0}\right)+K_{1} E\left[\sum_{i=0}^{\tau^{N}-1} 1_{\left(h_{i} \in C\right)}\right]
$$

Thus, $\rho E\left[\tau^{N}-1+1\right] \leq V\left(h_{0}\right)+K_{1}$, and by the Monotone Convergence Theorem,

$$
\rho \lim _{N \rightarrow \infty} E\left[\tau^{N}\right]=\rho E[\tau] \leq V\left(h_{0}\right)+K_{1}=1+K_{1} .
$$

Hence,

$$
E\left[\tau_{z+1}-\tau_{z}\right] \leq\left(1+K_{1}\right) / \rho
$$

uniformly for all $e_{\tau_{z}}, h_{\tau_{z}} \in C_{e}^{\prime} \times C_{h}$ values. Once perfect-zooming occurs, that is $h_{t} \in C_{h}$, then we have

$$
E\left[e_{t+1}^{2} \mid h_{t}, e_{t}\right] \leq\left(a^{2} / 2^{2 R}\right) \frac{\Delta_{t}^{2}}{4}+E\left[d_{t}^{2}\right]
$$

By the strong Markov property $\left(e_{\tau_{z}}, h_{\tau_{z}}\right)$ is also a Markov chain as $\left\{\tau_{z}<n\right\} \in \mathcal{F}_{n}$, the filtration at time $n$ for any $n \geq \tau_{z}$. The probability that $\tau_{z+1} \neq \tau_{z}+1$, is upper bounded by the probability:

$$
\begin{gathered}
P_{e}\left(\Delta_{\tau_{z}}\right):= \\
P\left(d_{\tau_{z}}^{2}>\left(\Delta_{\tau_{z}}(|a| / 2)\left(\frac{\lceil|a|+\epsilon\rceil-|a|-\epsilon+\eta}{|a|+\epsilon-\eta}\right)\right)^{2}\right)
\end{gathered}
$$

If $\tau_{z+1} \neq \tau_{z}+1$, then this means that the error is increasing and the system is once-again under-zoomed: $e_{\tau_{z}+1}=a e_{\tau_{z}}+d_{\tau_{z}}$ and $\Delta_{\tau_{z}+1}=\frac{|a|}{|a|+\epsilon-\eta} \Delta_{\tau_{z}}$. With some probability, the quantizer will still be in the perfect-zoom phase $\tau_{z+1}=\tau_{z}+1$. In case perfect-zoom is lost, there is a uniform bound on when the zoom is expected to be recovered. It follows that, conditioned on increment in the error, until the next stopping time, the process will increase exponentially and hence

$$
e_{\tau_{z+1}}=a^{\tau_{z+1}-\tau_{z}}\left(e_{\tau_{z}}+\sum_{t=0}^{\tau_{z+1}-\tau_{z}-1} a^{-t-1} d_{t+\tau_{z}}\right) .
$$

We now show that, there exist $\phi>0,|G|<\infty$ such that

$$
E\left[\log \left(e_{\tau_{z+1}}^{2}\right) \mid e_{\tau_{z}}\right] \leq(1-\phi) \log \left(e_{\tau_{z}}^{2}\right)+G 1_{\mid e_{\tau_{z} \mid \leq F}}
$$

After some relatively tedious steps, we obtain the following:

$$
\begin{aligned}
& E\left[\log \left(e_{\tau_{z+1}}^{2}\right) \mid e_{\tau_{z}}\right] \\
& \leq\left(1-P_{e}\left(\Delta_{\tau_{z}}\right)\right)\left(2 \log \left(|a| / 2^{R}\right)+\log \left(e_{\tau_{z}}^{2}\right)\right) \\
& \quad+P_{e}\left(\Delta_{\tau_{z}}\right)\left(2 E\left[\left(\tau_{z+1}-\tau_{z}\right)\right] \log (|a|)\right. \\
& \left.\quad+\log \left(2(e)_{\tau_{z}}^{2}+2 \frac{E\left[d^{2}\right]}{\left(1-|a|^{-1}\right)^{2}}\right)\right)
\end{aligned}
$$

We now proceed to upper-bound $E\left[\log \left(e_{\tau_{z+1}}^{2}\right) \mid e_{\tau_{z}}\right]$. Toward this end, we have

$$
\begin{aligned}
& P\left(\left|d_{\tau_{z}}\right|>\left(\Delta_{\tau_{z}}(|a| / 2)\left(\frac{\lceil|a|+\epsilon]-|a|-\epsilon+\eta}{|a|+\epsilon-\eta}\right)\right)\right) \\
\leq & \frac{E\left[d_{\tau_{z}}^{2}\right]}{\left(\Delta_{\tau_{z}}(|a| / 2)\left(\frac{\llbracket|a|+\epsilon|-| a \mid-\epsilon+\eta}{|a|+\epsilon-\eta}\right)\right)^{2}} \leq \frac{E\left[d_{\tau_{z}}^{2}\right]}{\left(K_{2} \Delta_{\tau_{z}}\right)^{2}},
\end{aligned}
$$

where we use Markov's inequality, with

$$
K_{2}=(|a| / 2)\left(\frac{\lceil|a|+\epsilon\rceil-|a|-\epsilon+\eta}{|a|+\epsilon-\eta}\right),
$$

and it follows that $P_{e}\left(\Delta_{\tau_{z}}\right) \leq \frac{E\left[d_{\tau_{z}}^{2}\right]}{\left(K_{2} \Delta_{\tau_{z}}\right)^{2}}$. Now, for large $e_{\tau_{z}}$ values, by substituting the uniform bound in (6), if the following holds, there is a drift to $C_{e}^{\prime}$. As $\log (1+$ $a) \leq a$ for $a \geq 0$, it suffices that the following holds for (7)

$$
\begin{gathered}
P_{e}\left(\Delta_{\tau_{z}}\right)\left\{2\left(1+K_{1}\right) / \rho \log (|a|)+\log (2)\right. \\
\left.+\frac{\frac{E\left[d^{2}\right]}{\left(1-|a|^{-1}\right)^{2}}}{e_{\tau_{z}}^{2}}+2 \log \left(2^{R} /|a|\right)\right\} \\
<2 \log \left(2^{R} /|a|\right)
\end{gathered}
$$

Let

$$
\begin{aligned}
I=\left(2\left(1+K_{1}\right) / \rho\right) \log (|a|)+\log (2) & +2 \log \left(2^{R} /|a|\right) \\
& +\frac{E\left[d^{2}\right]}{\left(1-|a|^{-1}\right)^{2}}
\end{aligned}
$$

Then, it follows that, for $2 \log \left(2^{R} /|a|\right)-\lambda>0$

$$
F=\max \left(1 / K_{2}, \sqrt{\frac{E\left[d_{t}^{2}\right] I}{K_{2}^{2}\left(2 \log \left(\frac{2^{R}}{\mid a}\right)-\lambda\right)}}\right)>0,
$$

the following holds for some $\lambda>0$ :

$$
E\left[\log \left(e_{\tau_{z+1}}^{2}\right)\right] \leq(1-\lambda) \log \left(e_{\tau_{z}}^{2}\right)+G 1_{\left|\left(e_{\tau_{z}}\right)\right| \leq F}
$$


with $\left.G=2\left(1+K_{1}\right) / \rho\right) \log |a|+\log \left(2 F^{2}+2 \frac{E\left[d^{2}\right]}{\left(1-|a|^{-1}\right)^{2}}\right)$ $+2 \log \left(\frac{2^{R}}{|a|}\right)$. Hence, we have obtained another drift condition for the sampled Markov chain. This shows that the newly constructed Markov process $e_{\tau_{z}}$ hits $C_{e}^{\prime}=\left\{e_{t}:\left|e_{t}\right| \leq F\right\}$ infinitely often. Let us call this process $\tau_{y}$, and define

$$
\begin{gathered}
\tau_{y+1}=\inf \left\{t>\tau_{y}:\left|e_{t}\right| \leq F,\left|h_{t}\right| \leq 1 \mid\right\} \\
=\inf \left\{\tau_{z+k}>\tau_{z}:\left|e_{\tau_{z+k}}\right| \leq F\right\}
\end{gathered}
$$

Hence, $k$ is the number of visits such that $\{h:|h(t)| \leq$ $1\}$ until $e_{t}$ hits $C_{e}^{\prime}$. In this set the cost is finite. When there is an excursion outside this set, the expected length of the trip (in terms of the new Markov process) is finite, that is $E\left[\tau_{y+1}-\tau_{y}\right]<\infty$. This follows because of the following: Define a variable $M_{\tau_{z}}:=\log \left(e_{\tau_{z}}^{2}\right)$, and for $k \geq 1, M_{\tau_{z+k}}=\log \left(e_{\tau_{z+k}}^{2}\right)-\sum_{l=0}^{k-1}(1-\lambda) \log \left(e_{\tau_{z+l}}^{2}\right)-$ $G 1_{\left|\left(e_{\tau_{z}+l}\right)\right| \leq F}, M_{\tau_{z+k}}$ is a Super-Martingale sequence since $E\left[M_{\tau_{z+1}} \mid \mathcal{F}_{\tau_{z}}\right] \leq M_{\tau_{z}}$. For any finite $n$, let us define $k^{n}=\min \left(k \leq n, k+\log \left(e_{\tau_{z}+l}^{2}\right) \geq n\right)$, which is a stopping time. Hence,

$$
E\left[\sum_{l=0}^{k^{n}-1}(1-\lambda) \log \left(e_{\tau_{z+l}}^{2}\right)\right] \leq M_{0} \leq \log \left(F^{2}\right)
$$

Since $\log \left(e_{\tau_{z+l}}^{2}\right) \geq E\left[\tau_{z+l+1}-\tau_{z+l}\right]$ for $F$ large enough, it follows that:

$$
(1-\lambda) E\left[\sum_{l=1}^{k^{n}} \tau_{z+l}-\tau_{z+l-1}\right] \leq M_{0} \leq \log \left(F^{2}\right)
$$

or $E\left[\tau_{z+k^{n}}-\tau_{z}\right] \leq M_{0} \leq \frac{\log \left(F^{2}\right)}{(1-\lambda)}$. Finally, taking the limit as $n \rightarrow \infty$, and by the Monotone Convergence Theorem, it follows that $E\left[\tau_{(z+k)}-\tau_{z}\right] \leq \frac{\log \left(F^{2}\right)}{(1-\lambda)}$ and

$$
\sup _{\left(e_{\tau_{z}}, h_{\tau_{z}}\right) \in C_{e}^{\prime} \times C_{h}} E\left[\tau_{(z+k)}-\tau_{z}\right] \leq \frac{\log \left(F^{2}\right)}{(1-\lambda)}
$$

This leads to a finite expected cost in the logarithm of the magnitude.

\section{Proof of Theorem 2.2: EXISTENCE OF AN INVARIANT PROBABILITY DISTRIBUTION}

Before proceeding further, we review a number of definitions useful for the development in this section. Let $\left\{x_{t}, t \geq 0\right\}$ be a Markov chain with state space $(\mathbb{X}, \mathcal{B}(\mathbb{X})$, and defined on a probability space $(\Omega, \mathcal{F}, \mathcal{P})$, where $\mathcal{B}(\mathbb{X})$ denotes the Borel $\sigma$-field on $\mathbb{X}, \Omega$ is the sample space, $\mathcal{F}$ a sigma field of subsets of $\Omega$, and $\mathcal{P}$ a probability measure. Let $P(x, D):=P\left(x_{t+1} \in D \mid x_{t}=\right.$ $x$ ) denote the transition probability from $x$ to $D$, that is the probability of the event $\left\{x_{t+1} \in D\right\}$ given that $x_{t}=x$.
Definition 3.1: For a Markov chain with transition probability defined as before, a probability measure $\pi$ is invariant on the Borel space $(\mathbb{X}, \mathcal{B}(\mathbb{X}))$ if

$$
\pi(D)=\int_{\mathbb{X}} P(x, D) \pi(d x), \quad \forall D \in \mathcal{B}(\mathbb{X}) .
$$

Definition 3.2: A Markov chain is $\mu$-irreducible, if for any set $B \subset \mathbb{X}$, such that $\mu(B)>0$, and $\forall x \in \mathbb{R}$, there exists some integer $n>0$, possibly depending on $B$ and $x$, such that $P^{n}(x, B)>0$, where $P^{n}(x, B)$ is the transition probability in $n$ stages, that is $P\left(x_{t+n} \in\right.$ $\left.B \mid x_{t}=x\right)$.

Definition 3.3: [12] A set $A \subset \mathbb{X}$ is $\mu$-petite on $(\mathbb{X}, \mathcal{B}(\mathbb{X})$ ) if for some distribution $\mathcal{T}$ on $\mathbb{N}$ (set of natural numbers), and some non-trivial probability measure $\mu$, $\sum_{n=0}^{\infty} P^{n}(x, B) \mathcal{T}(n) \geq \mu(B), \quad \forall x \in A$, and $B \in \mathcal{B}(\mathbb{X})$, where $\mathcal{B}(\mathbb{X})$ denotes the (Borel) sigma-field on $\mathbb{X}$.

Theorem 3.1: ([12] Thm. 4.1) Consider a Markov process $\left\{x_{t}\right\}$ taking values in $\mathbb{X}$. A compact set $A \in \mathbb{X}$, is recurrent if $P\left(\min \left(t>0: x_{t} \in A\right)<\infty \mid x_{0}=\right.$ $x)=1, \forall x \in \mathbb{X}$. If the recurrent set $A$ is a $\mu$-petite set, if the chain is $\mu$-irreducible, and if $\sup _{x \in A} E[\min (t>$ $\left.\left.0: x_{t} \in A\right) \mid x_{0}=x\right]<\infty$, then the Markov chain is positive Harris recurrent and it admits a unique invariant distribution.

In our setting, $\left(e_{t}, \Delta_{t}\right)$ forms the Markov chain. In view of the above results, we now show that the set of bin sizes forms a communication class under the hypothesis of the theorem: Since we have $\Delta_{t+1}=\bar{Q}\left(\left|\frac{e_{t}}{\Delta_{t} 2^{R}}\right|\right) \Delta_{t}$, it follows that

$$
\log _{2}\left(\Delta_{t+1}\right) / s=\log _{2}\left(\bar{Q}\left(\left|\frac{e_{t}}{\Delta_{t} 2^{R}}\right|\right)\right) / s+\log _{2}\left(\Delta_{t}\right) / s,
$$

is also an integer. Furthermore, since the source process $x_{t}$ is Lebesgue-irreducible (as the system noise admits a continuous probability density function), and there is a uniform lower bound on the bin-size $L^{\prime}$, the error process takes values in any of the admissible quantizer bins with non-zero probability. Let the values taken by $\log _{2}\left(\bar{Q}\left(\left|\frac{e_{t}}{\Delta_{t} 2^{R}}\right|\right)\right) / s$ be $\{-A, B\}$. By the hypothesis of the theorem statement, $A, B$ are relatively prime. Consider two integers $k, l \geq \frac{\log _{2}\left(L^{\prime}\right)}{s}$. Further, assume, without any loss of generality that $l>k$. From $k$ to $l$, one can construct a sequence consisting of $-A$ and $B$ integers such that the sum of these integers equals $l-k$ for all $l, k \in \mathbb{N}$, that is there exist $N_{A}, N_{B} \in \mathbb{Z}_{+}$such that

$$
l-k=-N_{A} A+N_{B} B .
$$

Consider first the case where $k>\frac{\log _{2}\left(L^{\prime}\right)}{s}+N_{A} A$. We show that the probability of $N_{A}$ occurrences of perfect 
zoom, and $N_{B}$ occurrences of under-zoom phases is bounded away from zero. This set of occurrences includes the event that in the first $N_{A}$ time stages perfectzoom occurs and later, successively, $N_{B}$ times underzoom phase occurs. The probability of this event is lower bounded by $\left(P\left(d_{t} \in\left[-|a| 2^{s k}-L^{\prime},-|a| 2^{s k}+\right.\right.\right.$ $\left.\left.\left.\left.L^{\prime}\right]\right)\right)^{N_{A}}\left(P\left(d_{t}>|a| 2^{s l}\right]\right)\right)^{N_{B}}>0$. A similar analysis can be performed when $k<\frac{\log _{2}\left(L^{\prime}\right)}{s}+N_{A} A$, by considering the opposite order of events, where in the first $N_{B}$ times, under-zoom occurs, and in the successive $N_{A}$ time stages, perfect-zoom occurs. As such, the selection of these events will always have non-zero probability due to the Lebesgue irreducibility of the noise distribution. For any two admissible integers $k, l$ for some $p>0, P\left(\log _{2}\left(\Delta_{t+p}\right)=l s \mid \log _{2}\left(\Delta_{t}\right)=k s\right)>$ 0 . Now, we can connect the results of the previous section with Theorem 3.1. The recurrent set $C_{e}^{\prime} \times C_{h}$ is $\nu$-petite, for some probability measure $\nu$ as any set in the state space is visited starting from $C_{e}^{\prime} \times C_{h}$, and the chain is irreducible. These two imply that the chain is positive Harris recurrent.

\section{Simulation}

As a simulation study, we consider a linear system with the following dynamics: $x_{t+1}=2.5 x_{t}+u_{t}+d_{t}$, where $E\left[d_{t}\right]=0, E\left[d_{t}^{2}\right]=1$, and $\left\{d_{t}\right\}$ are i.i.d. Gaussian variables. We use the zooming quantizer with rate $\log _{2}(4)=2$, since 4 is the smallest integer as large as $\lceil 2.5\rceil+1$. We have taken $L^{\prime}=1$.

The plot below (Figure 2) corroborates the stochastic stability result, by explicitly showing the under-zoomed and perfectly zoomed phases, with the peaks in the plots showing the under-zoom phases.

\section{CONCLUSiOn}

We provided a stochastic stability result for the zooming quantizers. In particular, we showed that zooming quantizers lead to a weak form of stability and are efficient.

\section{REFERENCES}

[1] D. J. Goodman and A. Gersho, "Theory of an adaptive quantizer, IEEE Trans. Commun., vol. 22, pp. 1037- 1045, Aug. 1974.

[2] J. C. Kieffer, "Stochastic stability for feedback quantization schemes", IEEE Trans. Inform. Theory, vol. 28, pp. 248-254, March 1982.

[3] J. C. Kieffer and J. G. Dunham "On a type of stochastic stability for a class of encoding schemes", IEEE Trans. Inform. Theory, vol. 29, pp. 793-797, November 1983.

[4] D. Liberzon, "On stabilization of linear systems with limited information", IEEE Trans. on Automatic Control, vol. 48, pp.
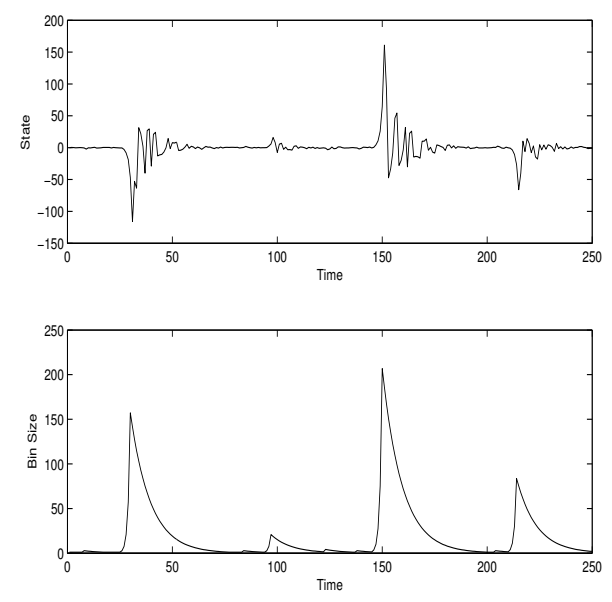

Fig. 2: Time horizon is 250 .

[5] D. Liberzon and D. Nesic, "Input-to-state stabilization of linear systems with quantized state measurements", IEEE Trans. on Automatic Control, vol. 52, no. 5, pp. 767-781, May 2007.

[6] R. Brockett and D. Liberzon, "Quantized feedback stabilization of linear systems",IEEE Trans. on Automatic Control, vol. 45, pp. 1279-1289, July 2000.

[7] W. S. Wong and R. W. Brockett, "Systems with finite communication bandwidth constraints - part II: Stabilization with limited information feedback, IEEE Trans. Automatic Control, 42(9):1294-1299, September 1997.

[8] S. Yüksel, "Optimal LQG coding and control over communication channels: An existence and an infeasibility result", in Proc. IEEE American Control Conf, Seattle, Washington, June 2008.

[9] V. S. Borkar, S. K. Mitter, and S. Tatikonda "Optimal sequential vector quantization of Markov sources," SIAM J. Control and Optimization, vol. 40, pp. 135-148, 2001.

[10] G. N. Nair and R. J. Evans, "Stabilizability of stochastic linear systems with finite feedback data rates", SIAM Journal on Control and Optimization, vol. 43, pp. 413 - 436, July 2004.

[11] R. M. Gray and D. L. Neuhoff, "Quantization," IEEE Trans. Inform. Theory, 44: 2325-2384, October 1998.

[12] S. P. Meyn and R. Tweedie, "Stability of Markovian processes I: Criteria for discrete-time chains", Adv. Appl. Probability, vol. 24, pp. 542-574, 1992. 